\title{
Symmetrisation in the Semiclassical Theory of Coulomb Excitation
}

\author{
A.J. Sargeant, R.C. Johnson and J.A. Tostevin ${ }^{1}$ \\ ${ }^{1}$ Department of Physics, University of Surrey, Guildford, GU2 5XH, U.K.
}

(Dated: November 5, 2011)

\begin{abstract}
Corrections to the Alder and Winther semiclassical cross section for first order Coulomb excitation are considered through the Sukumar and Brink systematic expansion of the propagator in inverse powers of Sommerfeld's parameter, $\eta$. The zeroth order term is that provided by unsymmetrised Alder-Winther theory and it is shown that to order $1 / \eta$ the only type of correction is one corresponding to energy symmetrisation.
\end{abstract}

PACS numbers: PACS numbers: 24.10.-i, 25.70.De, 25.70.Bc 


\section{INTRODUCTION}

Semiclassical theories have been used successfully for more than forty years (see ref. [1] and references therein) to describe Coulomb excitation; providing qualitative pictures of the physics and relatively simple quantitative methods. The standard semiclassical theory of Coulomb excitation, largely due to Alder and Winther [1], describes the relative motion of the projectile and target by a Rutherford orbit (the classical path for elastic scattering) whilst treating the excitation caused by their residual interaction quantum mechanically. This is thought to be a valid picture if Sommerfeld's dimensionless parameter

$$
\eta=\frac{Z_{t} Z_{p} e^{2}}{\hbar v_{0}}
$$

is large compared to unity. $Z_{t}$ and $Z_{p}$ are the charges of the target and projectile respectively and $v_{0}$ their asymptotic incident relative velocity in the centre of mass frame.

More recently Sukumar and Brink [2] have developed a systematic semiclassical theory of inelastic scattering by making an expansion of Feynman's path integral expression for the full quantum mechanical propagator about the classical paths for elastic scattering. The expansion parameter for the case of Coulomb excitation is $1 / \eta$. To zeroth order the theory leads to identical results to those obtained in Alder and Winther's approach with unsymmetrised orbits. Sukumar and Brink show how to calculate all leading order corrections in their $1 / \eta$ expansion.

In this paper we use the Sukumar and Brink method to calculate the unpolarised differential cross section to leading order in the coupling potential. For this case it is well known (see e.g. Chapter 10 of Ref. [1]) that when an energy symmetrisation prescription is used, Alder-Winther theory agrees well with exact quantum mechanical calculations. Alder and Winther assert that the only leading order correction, in $1 / \eta$, to the simplest unsymmetrised semiclassical theory corresponds to energy symmetrisation. They suggest a proof of this only for the case of dipole excitation.

We show that energy symmetrisation has a natural justification in terms of the Sukumar and Brink expansion. Further, the present approach shows that for the leading order unpolarised differential cross section, there are no other corrections of order $1 / \eta$. For example, there is no $1 / \eta$ correction corresponding to angular momentum symmetrisation. 


\section{UNPOLARISED DIFFERENTIAL CROSS SECTION}

Following Sukumar and Brink the unpolarised inelastic differential cross section for a transition from initial internal state $|\nu\rangle=\left|I_{\nu}, M_{\nu}\right\rangle$ to final internal state $|\sigma\rangle=\left|I_{\sigma}, M_{\sigma}\right\rangle$ is given by [3]

$$
\frac{d \sigma_{\sigma \nu}}{d \Omega}\left(E_{0}, \theta_{S}\right)=\frac{v_{1}}{v_{0}} \frac{d \sigma_{R}}{d \Omega}\left(E_{0}, \theta_{S}\right) \frac{1}{2 I_{\nu}+1} \sum_{M_{\sigma} M_{\nu}}\left(\left|a_{\sigma \nu}\left[\boldsymbol{r}_{R}(t)\right]\right|^{2}+2 \operatorname{Re}\left(a_{\sigma \nu}^{*} \delta a_{\sigma \nu}\right)\right) .
$$

The leading term in Eq. (2) is that obtained from unsymmetrised Alder-Winther theory and the second term is the correction of order $1 / \eta$. The final state relative velocity is $v_{1}$, and we have averaged over the initial and summed over the final spin projections. In Eq. (2) the Rutherford cross section is given by

$$
\frac{d \sigma_{R}}{d \Omega}\left(E_{0}, \theta_{S}\right)=\frac{1}{4 a_{0}^{2}} \csc ^{4}\left(\theta_{S} / 2\right)
$$

where $E_{0}$ is the incident relative energy and $\theta_{S}$ the centre of mass scattering angle. The quantity $a_{0}$ is half the distance of closest approach in a head on collision and is given by

$$
a_{0}=\frac{Z_{t} Z_{p} e^{2}}{m v_{0}^{2}}
$$

where $m$ denotes the reduced mass of the target and projectile.

Considering electric excitations to first order, the matrix element $a_{\sigma \nu}\left[\boldsymbol{r}_{R}(t)\right]$ which is a functional of the Rutherford orbit, $\boldsymbol{r}_{R}(t)$, decomposes into a sum over multipolarities $\lambda, \mu$ [1] so that[7].

$$
a_{\sigma \nu}=\frac{4 \pi Z_{t} e}{i \hbar} \sum_{\lambda \mu} \frac{1}{2 \lambda+1}\left(I_{\nu} M_{\nu} \mid I_{\sigma} M_{\sigma} \lambda \mu\right)\left\langle I_{\nu}|| \mathcal{M}(E \lambda)|| I_{\sigma}\right\rangle S_{E \lambda \mu}
$$

where in Alder and Winther's coordinate system B

$$
S_{E \lambda \mu}=v_{0}^{-1} a_{0}^{-\lambda} \sum_{\mu^{\prime}} \mathcal{D}_{\mu \mu^{\prime}}^{\lambda}\left(0,-\frac{\pi}{2}, 0\right) Y_{\lambda \mu^{\prime}}\left(\frac{\pi}{2}, 0\right) I_{\lambda \mu^{\prime}}\left(\theta_{S}, \xi\right)
$$

Here $I_{\lambda \mu}\left(\theta_{S}, \xi\right)$ is an orbital integral which is a function of the scattering angle and the dimensionless adiabaticity parameter

$$
\xi=\eta \frac{\Delta E}{2 E_{0}}
$$

with $\Delta E$ the excitation energy. 
Dos Aidos et al. [5] show that the correction to the amplitude $a_{\sigma \nu}$, required in the second term of Eq. (2), can be written to first order as a sum of terms

$$
\delta a_{\sigma \nu}=\Delta_{1}+\Delta_{2}+\Delta_{3}+\Delta_{4}+\Delta_{5} .
$$

We examine each of these contributions in turn.

(i) The first term is given by

$$
\begin{aligned}
\Delta_{1} & =-\frac{\Delta E}{2}\left(\frac{\partial}{\partial E_{0}}\right)_{\theta_{0}} a_{\sigma \nu} \\
& =\frac{3}{2} \frac{\xi^{2}}{\eta}\left(\frac{\partial}{\partial \xi}\right)_{\theta_{0}} a_{\sigma \nu},
\end{aligned}
$$

where we have used the result

$$
\left(\frac{\partial \xi}{\partial E_{0}}\right)_{\Delta E}=-\frac{3}{2} \frac{\xi}{E_{0}}
$$

to re-express the derivative with respect to $E_{0}$ as a derivative with respect to $\xi$ and make explicit the $1 / \eta$ dependence of the correction. The derivative in Eq. (10) acts only on the orbital factor $S_{E \lambda \mu}$. Noting that

$$
\frac{3}{2} \frac{\xi^{2}}{\eta}\left(\frac{\partial}{\partial \xi}\right)_{\theta_{0}}\left(\frac{1}{v_{0} a_{0}^{\lambda}}\right)=-\left(\lambda-\frac{1}{2}\right) \frac{\xi}{\eta}\left(\frac{1}{v_{0} a_{0}^{\lambda}}\right),
$$

we see that

$$
\begin{aligned}
\frac{3}{2} \frac{\xi^{2}}{\eta}\left(\frac{\partial}{\partial \xi}\right)_{E_{0}} S_{E \lambda \mu} & =-\left(\lambda-\frac{1}{2}\right) \frac{\xi}{\eta} S_{E \lambda \mu} \\
& +\frac{3}{2} \frac{\xi^{2}}{\eta} v_{0}^{-1} a_{0}^{-\lambda} \sum_{\mu^{\prime}} \mathcal{D}_{\mu \mu^{\prime}}^{\lambda}\left(0,-\frac{\pi}{2}, 0\right) Y_{\lambda \mu^{\prime}}\left(\frac{\pi}{2}, 0\right)\left(\frac{\partial}{\partial \xi}\right)_{\theta_{0}} I_{\lambda \mu^{\prime}}\left(\theta_{S}, \xi\right) .
\end{aligned}
$$

(ii) The term

$$
\begin{aligned}
\Delta_{2} & =\frac{1}{E_{0}} a_{\sigma \nu} \frac{\Delta E}{2} \\
& =\frac{\xi}{\eta} a_{\sigma \nu} .
\end{aligned}
$$

(iii) It is evident that

$$
\Delta_{3}=-\frac{i \hbar}{4 \eta}\left(\cot \theta_{0} \frac{\partial}{\partial \theta_{0}}+\cos ^{2} \theta_{0} \frac{\partial^{2}}{\partial \theta_{0}^{2}}\right)_{E_{0}} a_{\sigma \nu},
$$


where $\theta_{0}=\frac{1}{2}\left(\pi-\theta_{S}\right)$, does not contribute to the cross section to first order in the coupling because $a_{\sigma \nu},\left(\frac{\partial}{\partial \theta_{0}}\right)_{E_{0}} a_{\sigma \nu}$ and $\left(\frac{\partial^{2}}{\partial \theta_{0}^{2}}\right)_{E_{0}} a_{\sigma \nu}$ are all purely imaginary. $\Delta_{3}$ may however contribute to other observables (see ref. [6] for evaluation of the $1 / \eta$ contribution to the leading order tensor analysing powers for elastic scattering) and to the unpolarised cross section when terms of higher order than first in the coupling are included in $a_{\sigma \nu}$ (ref. [5] discusses multiple excitation of a rotational band).

(iv) Similarly

$$
\Delta_{4} \propto i a_{\sigma \nu}
$$

and does not contribute to the cross section to any order.

(v) Finally

$$
\Delta_{5}=\frac{i}{4 \eta}\left(2 i \xi \sin \theta_{0} \cos \theta_{0} \frac{\partial}{\partial \alpha}+\cos ^{2} \theta_{0} \frac{\partial^{2}}{\partial \alpha^{2}}+\frac{\partial^{2}}{\partial \beta^{2}}-\cot ^{2} \theta_{0} \frac{\partial^{2}}{\partial \gamma^{2}}\right) a_{\sigma \nu},
$$

where $\alpha, \beta$ and $\gamma$ are angles specifying the orientation of the Rutherford orbit. It can be shown, from symmetry arguments, that $\Delta_{5}$ does not contribute to the cross section at any order [5] but may contribute to other observables [6].

There are further $1 / \eta$ corrections to the amplitude $a_{\sigma \nu}$ in addition to those considered above [5]. These additional corrections are at least second order in the coupling, do not contribute to the leading order inelastic cross section, and are not evaluated here.

Substituting Eq.'s (5) and (8) into Eq. (2) and noting relation (13), the sum over spin projections may be performed in the usual manner to obtain

$$
\frac{d \sigma_{\sigma \nu}}{d \Omega}\left(E_{0}, \theta_{S}\right)=\frac{p_{1}}{p_{0}}\left(\frac{Z_{t} e}{\hbar v_{0}}\right)^{2} a_{0}^{-2 \lambda+2} B\left(E \lambda, I_{\nu} \rightarrow I_{\sigma}\right) \frac{d f_{E \lambda}}{d \Omega}\left(\theta_{S}, \xi\right),
$$

where

$$
\frac{d f_{E \lambda}}{d \Omega}\left(\theta_{S}, \xi\right)=\left(1-\frac{\xi}{\eta}\left((2 \lambda-3)-\frac{3}{2} \xi \frac{\partial}{\partial \xi}\right)\right)_{\theta_{0}} \frac{d f_{E \lambda}^{(0)}}{d \Omega}\left(\theta_{S}, \xi\right) .
$$

Here Alder and Winther's differential cross section function is given by

$$
\frac{d f_{E \lambda}^{(0)}}{d \Omega}\left(\theta_{S}, \xi\right)=\frac{4 \pi^{2}}{(2 \lambda+1)^{3}} \sum_{\mu}\left|Y_{\lambda \mu}\left(\frac{\pi}{2}, 0\right)\right|^{2}\left|I_{\lambda \mu}\left(\theta_{S}, \xi\right)\right|^{2} \csc ^{4} \frac{\theta_{S}}{2} .
$$

The first term in Eq. (20) is obtained from standard Alder-Winther theory with unsymmetrised orbits and the rest is the correction of order $1 / \eta$. As is usual in a first order theory the cross section separates into a factor depending on the orbital parameters and a factor $B\left(E \lambda, I_{\nu} \rightarrow I_{\sigma}\right)$, the reduced transition probability, describing internal excitation. The inclusion of the $1 / \eta$ term only modifies the orbital factor. 


\section{INTERPRETATION OF THE CORRECTION TERMS}

The correction may be attributed to the energy loss from the relative motion due to the excitation and it is interesting to compare this result with the energy symmetrisation procedure suggested by the WKB approximation for the relative motion wavefunctions [1]. This procedure consists of replacing those parameters dependent on the incident relative energy, in the zeroth order (in $1 / \eta$ ) expression for the differential cross section, by symmetrised versions which take account of the difference between the initial and final relative energies. Thus

$$
\frac{d \sigma_{\sigma \nu}^{S Y M}}{d \Omega}\left(E_{0}, \theta_{S}\right)=\frac{p_{1}}{p_{0}}\left(\frac{Z_{t} e}{\hbar v_{S Y M}}\right)^{2} a_{S Y M}^{-2 \lambda+2} B\left(E \lambda, I_{\nu} \rightarrow I_{\sigma}\right) \frac{d f_{E \lambda}^{(0)}}{d \Omega}\left(\theta_{S}, \xi_{S Y M}\right),
$$

where

$$
\begin{gathered}
v_{S Y M}=\left(v_{0} v_{1}\right)^{\frac{1}{2}}=v_{0}\left(1-\frac{2 \xi}{\eta}\right)^{\frac{1}{4}}, \\
a_{S Y M}=\frac{Z_{p} Z_{t} e^{2}}{m v_{S Y M}^{2}}
\end{gathered}
$$

and

$$
\xi_{S Y M}=\frac{Z_{p} Z_{t} e^{2}}{\hbar}\left(\frac{1}{v_{1}}-\frac{1}{v_{0}}\right)=\eta\left(1-\frac{2 \xi}{\eta}\right)^{-\frac{1}{2}}-\eta .
$$

Taylor expanding Eq. (22) about the unsymmetrised values we obtain precisely the right hand side of Eq. (19) plus additional terms of order $1 / \eta^{2}$.

\section{CONCLUSIONS}

We have evaluated the $1 / \eta$ corrections to the Alder and Winther semiclassical theory of Coulomb excitation, for the case of the unpolarised leading order differential cross section, using the Sukumar and Brink expansion of the propagator in powers of $1 / \eta$. The value of the Sukumar and Brink method is that it is systematic, allowing one to calculate all corrections to the standard theory up to a given order. For the particular case considered here we have shown that the only correction, to order $1 / \eta$, corresponds to energy symmetrisation. There may be further $1 / \eta$ corrections when other observables and higher order processes are considered. 


\section{Acknowledgments}

This research is supported by EPSRC, U.K., grant GR/J95867 and a research studentship.

[1] K. Alder and A. Winther, Electromagnetic Excitation, (North Holland Publ. Co., Amsterdam, 1975).

[2] C.V. Sukumar and D.M. Brink, Nucl. Phys. A404 (1983) 121.

[3] F.D. Dos Aidos and D.M. Brink, J. of Phys. G11 (1985) 249.

[4] D.M. Brink and G.R. Satchler, Angular Momentum, 3rd ed., (Clarendon Press, Oxford, 1993).

[5] F.D. Dos Aidos, C.V. Sukumar and D.M. Brink, Nucl. Phys. A448 (1986) 333.

[6] E.J. Roberts, C.V. Sukumar, R.C. Johnson and D.M. Brink, Nucl. Phys. A584 (1995) 362.

[7] We use Brink and Satchler's definitions [4] for reduced matrix elements and reduced transition probabilities. 